\title{
Exploring a SNR/molecular cloud association within HESS J1745-303
}

F. Aharonian ${ }^{1,13}$, A. G. Akhperjanian ${ }^{2}$, U. Barres de Almeida ${ }^{8, \star}$, A. R. Bazer-Bachi ${ }^{3}$, B. Behera ${ }^{14}$, M. Beilicke $^{4}$, W. Benbow ${ }^{1}$, K. Bernlöhr ${ }^{1,5}$, C. Boisson ${ }^{6}$, O. Bolz ${ }^{1}$, V. Borrel ${ }^{3}$, I. Braun ${ }^{1}$, E. Brion ${ }^{7}$, A. M. Brown ${ }^{8}$, R. Bühler ${ }^{1}$, T. Bulik ${ }^{24}$, I. Büsching ${ }^{9}$, T. Boutelier ${ }^{17}$, S. Carrigan ${ }^{1}$, P. M. Chadwick ${ }^{8}$, L.-M. Chounet ${ }^{10}$, A. C. Clapson ${ }^{1}$, G. Coignet ${ }^{11}$, R. Cornils ${ }^{4}$, L. Costamante ${ }^{1,28}$, M. Dalton ${ }^{5}$, B. Degrange ${ }^{10}$, H. J. Dickinson ${ }^{8}$, A. Djannati-Ataï ${ }^{12}$, W. Domainko ${ }^{1}$, L. O'C. Drury ${ }^{13}$, F. Dubois ${ }^{11}$, G. Dubus ${ }^{17}$, J. Dyks ${ }^{24}$, K. Egberts ${ }^{1}$, D. Emmanoulopoulos ${ }^{14}$, P. Espigat ${ }^{12}$, C. Farnier ${ }^{15}$, F. Feinstein ${ }^{15}$, A. Fiasson ${ }^{15}$, A. Förster ${ }^{1}$, G. Fontaine ${ }^{10}$, S. Funk ${ }^{29}$, M. Füßling ${ }^{5}$, Y. A. Gallant ${ }^{15}$, B. Giebels ${ }^{10}$, J. F. Glicenstein ${ }^{7}$, B. Glück ${ }^{16}$, P. Goret ${ }^{7}$, C. Hadjichristidis ${ }^{8}$, D. Hauser ${ }^{1}$, M. Hauser ${ }^{14}$, G. Heinzelmann ${ }^{4}$, G. Henri' ${ }^{17}$, G. Hermann ${ }^{1}$, J. A. Hinton ${ }^{25}$, A. Hoffmann ${ }^{18}$, W. Hofmann ${ }^{1}$, M. Holleran ${ }^{9}$, S. Hoppe ${ }^{1}$, D. Horns ${ }^{18}$, A. Jacholkowska ${ }^{15}$, O. C. de Jager ${ }^{9}$, I. Jung ${ }^{16}$, K. Katarzyński ${ }^{27}$, E. Kendziorra ${ }^{18}$, M. Kerschhaggl ${ }^{5}$, B. Khélifi ${ }^{10}$, D. Keogh ${ }^{8}$, Nu. Komin ${ }^{15}$, K. Kosack ${ }^{1}$, G. Lamanna ${ }^{11}$, I. J. Latham ${ }^{8}$, M. Lemoine-Goumard ${ }^{10}$, J.-P. Lenain ${ }^{6}$, T. Lohse ${ }^{5}$, J. M. Martin ${ }^{6}$, O. Martineau-Huynh ${ }^{19}$, A. Marcowith ${ }^{15}$, C. Masterson ${ }^{13}$, D. Maurin ${ }^{19}$, T. J. L. McComb ${ }^{8}$, R. Moderski ${ }^{24}$, E. Moulin ${ }^{7}$, M. Naumann-Godo ${ }^{10}$, M. de Naurois ${ }^{19}$, D. Nedbal ${ }^{20}$, D. Nekrassov ${ }^{1}$, S. J. Nolan ${ }^{8}$, S. Ohm ${ }^{1}$, J.-P. Olive ${ }^{3}$, E. de Oña Wilhelmi ${ }^{12}$, K. J. Orford ${ }^{8}$, J. L. Osborne ${ }^{8}$, M. Ostrowski ${ }^{23}$, M. Panter $^{1}$, G. Pedaletti ${ }^{14}$, G. Pelletier ${ }^{17}$, P.-O. Petrucci ${ }^{17}$, S. Pita ${ }^{12}$, G. Pühlhofer ${ }^{14}$, M. Punch ${ }^{12}$, B. C. Raubenheimer ${ }^{9}$, M. Raue $^{4}$,

S. M. Rayner ${ }^{8}$, M. Renaud ${ }^{1}$, J. Ripken ${ }^{4}$, L. Rob ${ }^{20}$, S. Rosier-Lees ${ }^{11}$, G. Rowel1 ${ }^{26}$, B. Rudak ${ }^{24}$, J. Ruppel ${ }^{21}$,

V. Sahakian ${ }^{2}$, A. Santangelo ${ }^{18}$, R. Schlickeiser ${ }^{21}$, F. M. Schöck ${ }^{16}$, R. Schröder ${ }^{21}$, U. Schwanke ${ }^{5}$, S. Schwarzburg ${ }^{18}$, S. Schwemmer ${ }^{14}$, A. Shalchi ${ }^{21}$, H. Sol ${ }^{6}$, D. Spangler ${ }^{8}$, Ł. Stawarz $^{23}$, R. Steenkamp ${ }^{22}$, C. Stegmann ${ }^{16}$, G. Superina ${ }^{10}$, P. H. Tam ${ }^{14}$, J.-P. Tavernet ${ }^{19}$, R. Terrier ${ }^{12}$, C. van Eldik ${ }^{1}$, G. Vasileiadis ${ }^{15}$, C. Venter ${ }^{9}$, J. P. Vialle ${ }^{11}$, P. Vincent ${ }^{19}$, M. Vivier ${ }^{7}$, H. J. Völk ${ }^{1}$, F. Volpe ${ }^{10,28}$, S. J. Wagner ${ }^{14}$, M. Ward ${ }^{8}$, A. A. Zdziarski ${ }^{24}$, and A. Zech ${ }^{6}$

(Affiliations can be found after the references)

Received 11 December 2007 / Accepted 20 February 2008

\section{ABSTRACT}

Aims. HESS J1745-303 is an extended, unidentified VHE (very high energy) gamma-ray source discovered using HESS in the Galactic Plane Survey. Since no obvious counterpart has previously been found in longer-wavelength data, the processes that power the VHE emission are not well understood.

Methods. Combining the latest VHE data with recent XMM-Newton observations and a variety of source catalogs and lower-energy survey data, we attempt to match (from an energetic and positional standpoint) the various parts of the emission of HESS J1745-303 with possible candidates. Results. Though no single counterpart is found to fully explain the VHE emission, we postulate that at least a fraction of the VHE source may be explained by a supernova-remnant/molecular-cloud association and/or a high-spin-down-flux pulsar.

Key words. gamma rays: observations - X-rays: general - Galaxy: general - ISM: cosmic rays - ISM: clouds

\section{Introduction}

The Galactic center region (roughly between $|l|<2^{\circ},|b|<1^{\circ}$ ) is densely populated with possible VHE emission candidates: supernova remnants (SNRs), dense molecular clouds, pulsar wind nebulae (PWNe), X-ray binaries (XRBs), and a variety of unidentified sources seen in lower wavebands. In this region, VHE emission has been detected from an as yet unidentified point-like source at the Galactic center (possibly associated with the super-massive black hole Sgr $A^{\star}$ or a PWN) (Tsuchiya et al. 2004; Kosack et al. 2004; Aharonian et al. 2004; Hinton \& Aharonian 2007), from the SNR G0.9+0.1 (Aharonian et al. 2005a) (point-like for HESS), and from a region of diffuse emission approximately $\pm 1^{\circ}$ in longitude, which

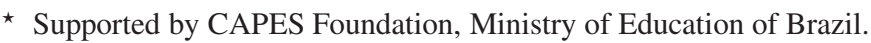

is most likely associated with the interaction of cosmic-ray particles with molecular clouds (Aharonian et al. 2006c), and finally HESS J1745-303, an extended, unidentified VHE gamma-ray source lying approximately a half degree below the Galactic plane at $l=-0.4$. HESS J1745-303 was first discovered in the HESS Galactic Plane Survey (Aharonian et al. 2006d). Subsequent observations of the region using HESS have provided increased exposure of this object, and thus a more detailed study is now possible.

VHE gamma rays are typically thought to be produced via two general mechanisms: the up-scatter of lower-energy photons by high-energy electrons via the inverse-Compton process, or the production and subsequent decay of $\pi^{0}$ s produced in the interactions of high-energy hadrons. Though it is difficult to distinguish between a purely leptonic or hadronic scenario in many 
of the currently published VHE sources, in cases where it is known that cosmic rays are interacting with a dense medium, the hadronic scenario becomes more viable. This is true for example on the Galactic center ridge, where the VHE emission is seen to roughly follow the location of dense molecular clouds (Aharonian et al. 2006c), or possibly in the case of SNRs embedded in dense regions of the interstellar medium (ISM) (e.g. Aharonian et al. 2007b, 2006e). The flux of gamma rays produced via the hadronic production of $\pi^{0} \mathrm{~s}$ depends linearly on the density of the surrounding medium. For typical Galactic SNRs (few kpc distance, age of $>1000 \mathrm{yrs}$, in a medium with average density $\sim 1 \mathrm{~cm}^{-3}$ ), the flux above $1 \mathrm{GeV}$ is predicted to be quite small (below the EGRET or even HESS sensitivity) (Aharonian et al. 1994). However, in the case where the supernova shock is interacting with a dense molecular cloud (as in the case of G359.1-0.5, discussed later), the emission can be significantly enhanced. Moreover, the expected flux in the TeV energy range from an interacting SNR shock may be much higher than the extrapolation of the spectrum measured in the $\mathrm{GeV}$ range (Aharonian \& Atoyan 1996).

Though the region around HESS J1745-303 is well covered by radio (e.g. VLA, LaRosa et al. 2000), and X-ray (e.g. ROSAT, Voges et al. 2000) observations, no obvious counterpart is visible that fully matches the morphology of this source. Here, we examine the possibility that HESS J1745-303 is (at least in part) associated with several counterpart candidates seen in other wavebands, most notably the interaction of a nearby supernova remnant with a molecular cloud. Additionally, we present an analysis of recent X-ray data from XMM-Newton covering the central part of this object.

\section{Technique}

\subsection{The HESS instrument}

HESS (the High Energy Stereoscopic System) is an array of four atmospheric Cherenkov telescopes (ACTs) located in the Khomas highlands of Namibia at an altitude of $1800 \mathrm{~m}$ above sea-level. Each telescope consists of a $107 \mathrm{~m}^{2}$ optical reflector made up of segmented spherical mirrors that focus light into a camera of 960 photo-multiplier tube pixels (Bernlöhr et al. 2003). Using the imaging atmospheric Cherenkov technique (e.g. Hillas 1985, 1996; Weekes 1996; Daum et al. 1997), the telescopes image the Cherenkov light emitted by the particles in extensive air showers from multiple viewpoints, and the energy and direction of the primary gamma ray can be reconstructed with an average energy resolution of $\sim 16 \%$ (above an energy threshold of approximately $150 \mathrm{GeV}$ ), and a spatial resolution of $\sim 0.1^{\circ}$ per event (Aharonian et al. 2006a). The large field of view $\left(\sim 5^{\circ}\right)$, and good off-axis sensitivity of the HESS array make it well suited for studying extended sources and for scan-based observations, where the source position is not known a priori.

\subsection{Data and analysis technique}

Following the standard HESS procedure, the data presented here are processed with separate analysis and calibration schemes: the HESS standard analysis (Aharonian et al. 2006a), in which showers are reconstructed and hadronic background is rejected via the Hillas moment-analysis technique (Hillas 1996); and the Model2D analysis described by de Naurois (2005), which employs a semi-analytic model of the shower to characterize each image. It should be noted that both the techniques and simulations used in them are independent, providing a robust check of the analysis. Since results of both analyses agree within errors, the results presented here are from the standard analysis only. The separation of gamma-ray candidates from cosmic-raylike events was made using both standard cuts (optimized for a lower energy threshold) and hard cuts (optimized for better background rejection) described in (Aharonian et al. 2006a), but with a larger angular angular integration radius to account for the extension of the source. The former were used for spectra, and the latter for producing the sky images, though both were checked for consistency.

The data for HESS J1745-303 are not primarily comprised of dedicated observations of the object, but rather from scanbased observations of the region (taken at regular grid-points along the galactic plane) and from dedicated observations of the VHE source at the Galactic center (HESS J1745-290), which lies approximately $1.4^{\circ}$ away. Due to the wide range of pointings and since the gamma-ray acceptance across the field of view of HESS falls off radially, the total exposure of the region around HESS J1745-303 is highly non-uniform; in particular there is a strong gradient toward the Galactic center, which can lead to increased systematic errors in background subtraction. To mitigate this situation, several different background selection techniques were employed in this analysis.

For the generation of two-dimensional images, both the fieldof-view background method, where the background for each observation is determined from a one-dimensional model of the radial acceptance (taken from observations with no significant emission in the field of view), and the ring background method, where the background at each point on the sky is calculated from an annulus surrounding it (again, with sources excluded), are used (Berge et al. 2007). Since the field-of-view background method is more sensitive to gradients in exposure, the images presented here employ the ring background method, though both methods are checked for reasonable (within 5\%) consistency.

For the spectral analysis background estimation, we use the reflected region technique (Berge et al. 2007), where background events are selected from circular off-source regions within the field of view. These regions are chosen with the same angular size and offset from the observation center position as the onsource integration region, ensuring their acceptance-corrected exposure is approximately equal to that of the on-source region. This technique is less suited to the generation of images, but for a known source position provides an estimate of the background that is independent of radial acceptance models.

To prevent contamination of the background from the diffuse gamma-ray emission and to avoid including emission from the various other sources near the Galactic center, regions around Sagittarius $A^{\star}$, around G $0.9+0.1$, as well as within $\pm 0.8^{\circ}$ of the Galactic plane, were not used for background estimation.

The statistical significances in both the images and the spectral analysis are calculated from the measured number of onand off-source (background) events following the likelihood ratio procedure outlined in Li \& Ma (1983).

Spectra are generated following the methods described by both Aharonian et al. (2006a), in the case of the standard analysis, and Piron et al. (2001) for the Model2D analysis. The on-source integration radius used for the generation of spectra is chosen (unless otherwise stated) to fully enclose the source (based on the radial profile of the signal and background), making no assumptions on the details of the source morphology and thus providing a less-biased result at the expense of a lower signal-to-noise ratio. Since the data set spans several years, during which the gain and optical efficiency of the telescopes has 
Table 1. Characteristics of the observations of HESS J1745-303 with HESS (Aharonian et al. 2006a) and XMM-Newton. The "full" dataset is what is presented here, while the 2006 subset was presented by Aharonian et al. (2006d). The energy range for the HESS case is the range used to fit the statistically significant spectral points; the full sensitivity of the detector extends to approximately $100 \mathrm{TeV}$.

\begin{tabular}{rccc}
\hline \hline & $\begin{array}{c}\text { HESS } \\
(2006)\end{array}$ & $\begin{array}{c}\text { HESS } \\
(\text { full })\end{array}$ & XMM-Newton \\
\hline Exposure time (h) & 35 & 79 & 4 \\
Angular Res. (arcmin) & 6 & 6 & 0.1 \\
Field-of-view ( $\left.{ }^{\circ}\right)$ & 5.0 & 5.0 & 0.5 \\
Energy range & $0.35-1.5 \mathrm{TeV}$ & $0.35-30 \mathrm{TeV}$ & $0.5-10 \mathrm{keV}$ \\
Energy Res. & $16 \%$ & $16 \%$ & $20-50 \%$ \\
\hline
\end{tabular}

changed, muon images are used to calibrate the energy estimate of each gamma-ray candidate (Aharonian et al. 2006a). The systematic error on the fluxes given here is estimated from simulated data to be $\pm 20 \%$ while the photon index has a typical systematic error of \pm 0.2 .

\section{VHE results}

With an exposure time of $35 \mathrm{~h}$, the original HESS data set presented by Aharonian et al. (2006d) revealed HESS J1745-303 at a pre-trials significance level of 6.3 standard deviations (for an integration radius of $0.224^{\circ}$, which is the HESS standard for blind source searches). With the increased exposure (now $79 \mathrm{~h}$ ) coming primarily from re-observations of the Galactic center source HESS J1745-290, HESS J1745-303 is now seen at well above the detection threshold, with a pre-trials significance level of 12 standard deviations $(\sigma)$.

Figure 1 shows an image of gamma-ray excess counts covering HESS J1745-303 with significance contours overlaid. The image is smoothed with a Gaussian kernel with standard deviation $0.07^{\circ}$ (chosen to reveal morphological features while maintaining good statistics), and the significance contours are generated with an oversampling radius of $0.12^{\circ}$, matched to the rms of the Gaussian smoothing to provide a visual impression of significant features. The source centroid is determined by fitting an elongated two-dimensional Gaussian convolved with the HESS point-spread function to the un-smoothed images. Due to the non-Gaussian morphology, this only gives a rough centroid of the emission.

For the spectral analysis, the integration region was taken to include the entire source (with a radius of $0.4^{\circ}$ centered on $17^{\mathrm{h}} 45^{\mathrm{m}} 2.10^{\mathrm{s}},-30^{\circ} 22^{\prime} 14.00^{\prime \prime}, \mathrm{J} 2000$ coordinates). For this larger integration region, we find a total significance of $10.2 \sigma$, with 2030 total excess counts. The spectrum, shown in Fig. 2, is well fit by a power law: $\mathrm{d} N / \mathrm{d} E=N_{0}(E / \mathrm{TeV})^{-\Gamma}$ with photon index $\Gamma=2.71 \pm 0.11_{\text {stat }} \pm 0.2_{\text {sys }}$, and a differential flux normalization $N_{0}$ of $\left(2.84 \pm 0.23_{\text {stat }} \pm 0.28_{\text {sys }}\right) \times 10^{-12} \mathrm{TeV}^{-1} \mathrm{~cm}^{-2} \mathrm{~s}^{-1}$. This corresponds to an integral flux above the peak energy of VHE events $(\sim 1 \mathrm{TeV})$ of $F(1-10 \mathrm{TeV})=(1.63 \pm 0.16) \times$ $10^{-12} \mathrm{~cm}^{-2} \mathrm{~s}^{-1}$. Figure 2 shows the resulting spectral energy distribution.

Figure 3 compares the new result to the data presented in Aharonian et al. (2006d). Below $700 \mathrm{GeV}$, the new result noticeably differs from the former spectrum, which was made with nearly a factor of 10 less photons (211 excess counts). Quantitatively, the new spectrum is $2.5 \sigma$ softer than the old photon index of $\Gamma=1.82 \pm 0.29_{\text {stat }} \pm 0.2_{\text {sys. }}$. Thus, the integral
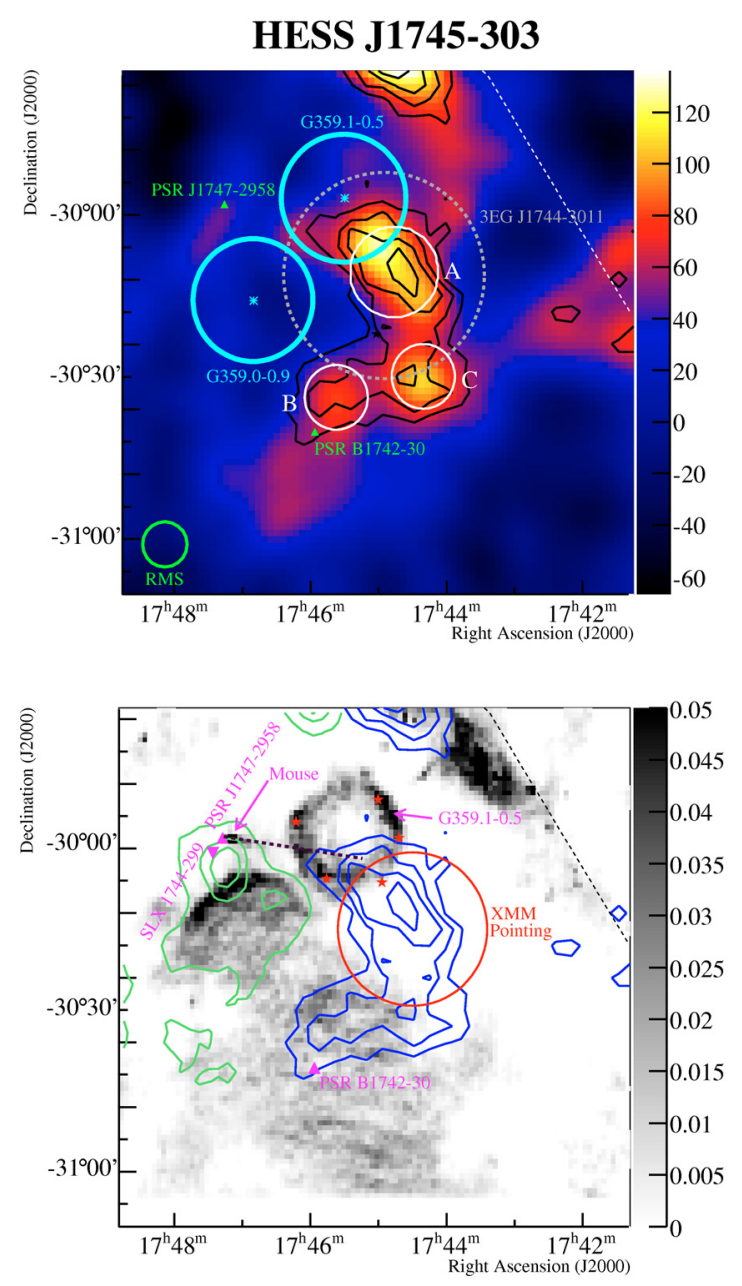

Fig. 1. Top: a VHE gamma-ray (excess count) image of HESS J1745303 with the positions of possible counterpart candidates overlaid for reference. The color scale set such that the blue/red transition occurs at the $\sim 3 \sigma$ (pre-trials) significance level. The $4 \sigma$ to $7 \sigma$ statistical significance contours are shown in black. The thin white circles represent the integration regions $\mathrm{A}, \mathrm{B}$, and $\mathrm{C}$ discussed in the text. The dashed circle is the 95\% error circle for the location of 3EG J1744-3011. The significant excess seen to the north is the tail end of the Galactic Ridge diffuse emission discussed by Aharonian et al. (2006c). The Galactic plane is marked with a dotted line. Bottom: the HESS significance contours (blue) overlaid on a VLA radio image (LaRosa et al. 2000) with overlaid ROSAT hard-band contours (green, Voges et al. 2000). Stars show the positions of $\mathrm{OH}$ masers, and the XMM-Newton field of view (Fig. 4) is drawn as a circle.

flux above $200 \mathrm{GeV}$ is smaller than the old value of $(11.2 \pm$ $\left.4.0_{\text {stat }} \pm 3.4_{\text {sys }}\right) \times 10^{-12} \mathrm{~cm}^{-2} \mathrm{~s}^{-1}$.

A re-analysis of only the data used in the original publication with our updated procedures gives a result that is in agreement with the one presented here; and a re-analysis of all other sources presented by Aharonian et al. (2006d) using the same techniques as in this paper gives results that are consistent with the original publication. Therefore, we have no indication that there is a general systematic error involved in either the old or the new analysis, but rather most likely a systematic error that exclusively (or predominantly) affects HESS J1745-303.

When analyzing an extended source that has both lowsurface-brightness and is located in a region of extremely uneven exposure, small uncertainties, e.g. in the acceptance-correction or background subtraction, may become significant. Such a problem only strongly affects low-surface-brightness sources 


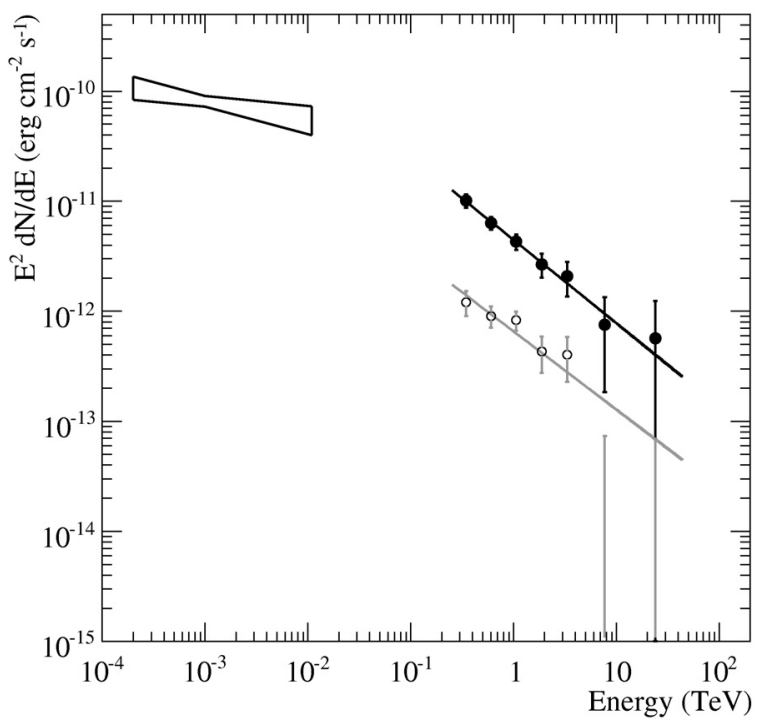

Fig. 2. The spectral energy distribution of VHE gamma rays from HESS J1745-303 (solid points) and from only region $A$ (open circles). The data are fit by a power law of the form $\mathrm{d} N / \mathrm{d} E=N_{0}(E / \mathrm{TeV})^{-\Gamma}$ see Sect. 3. The 3EG J1744-3011 flux is plotted as a bow-tie for comparison.

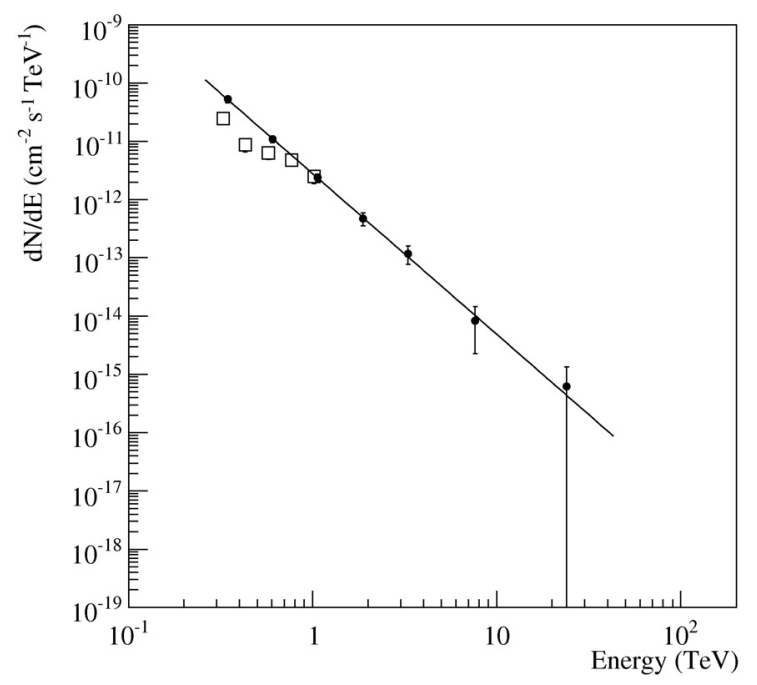

Fig. 3. Comparison of the spectra of HESS J1745-303 presented here (filled circles with line fit) with the previous result presented by Aharonian et al. (2006d) (open squares).

analyzed with large integration radii. For most HESS sources, wobble-mode observations are preformed that deliberately constrain systematic background errors to a minimum; but in the case of HESS J1745-303, the data set is dominated by observations of the Galactic center and the Galactic plane, therefore a uniform acceptance for all background control regions and the source region was probably not achieved in the old analysis to the required level. In contrast to the previous analysis, the large increase in observation time has provided a spectral-quality data set that is well above the detection threshold, over a much larger energy range, and that includes improvements in procedures used for the analysis of weak, extended sources. Here, we do not assume a particular source morphology and include time-dependent optical efficiency corrections to the energy, more exclusion regions for background subtraction (for sources that
Table 2. Integral fluxes of the three test regions A, B, and C (shown in Fig. 1), compared with the total integral flux from the full source. The photon index $\Gamma$ is derived from a fit to the spectrum for each region. Note that region A is chosen to correspond with the molecular cloud position and radius described in Sect. 5.1.

\begin{tabular}{llrc}
\hline \hline Region & $\begin{array}{c}F(1-10 \mathrm{TeV}) \\
\times 10^{-12} \mathrm{~cm}^{-2} \mathrm{~s}^{-1}\end{array}$ & $\%$ total & $\Gamma$ \\
\hline Full & $1.63 \pm 0.16$ & 100 & $2.71 \pm 0.11$ \\
A & $0.25 \pm 0.04$ & $15 \pm 3$ & $2.67 \pm 0.14$ \\
B & $0.11 \pm 0.02$ & $6 \pm 2$ & $2.93 \pm 0.21$ \\
C & $0.14 \pm 0.03$ & $8 \pm 2$ & $2.86 \pm 0.27$ \\
\hline
\end{tabular}

were subsequently discovered nearby), and software and lookuptable improvements that have reduced systematic errors due to uneven exposure.

Since the emission appears to have a complicated morphology with more than one peak in the excess image, the possibility that HESS J1745-303 is more than one source was explored. First, three emission peaks were determined, located at the positions A, B, and C shown in Fig. 1. Between each pair of peaks, a one-dimensional slice in the uncorrelated excess image (with a width of $0.1^{\circ}$ ) was made to determine the significance of the "dip" between them. In each case, the emission is no more than two standard deviations from a constant value across the slice. Furthermore, if the emission peaks are from multiple sources, one might expect to see spectral variability across the object, though energy dependent morphology may also arise from transport and/or energy-loss processes within a single source. To test this possibility, a spectral analysis was made at each of the test points with an integration radius of $0.14^{\circ}$ for $\mathrm{A}$ and $0.1^{\circ}$ for $\mathrm{B}$ and $\mathrm{C}$ (see Table 2). The spectral indices at each position are consistent with each other within statistical errors, and also with the spectrum determined for the entire source region; therefore within the statistics of the observations, there is no strong evidence to support the multiple-origin hypothesis.

\section{XMM-Newton observations of the region}

HESS J1745-303 was observed with the XMM-Newton X-ray satellite on September 18, 2006 for $30 \mathrm{ks}$ in satellite revolution 1241 (ObsID 0406580201). All X-ray instruments (EPIC MOS 1, MOS 2, and PN) were operated in full-frame mode and a medium filter was applied to screen out bright optical and UV sources. The calibration, data reduction and analysis made use of the XMM-Newton Science Analysis Software (SAS), version 7.0, together with the Extended Source Analysis Software package (XMM-ESAS), version 1.0 (Snowden et al. 2004). Following standard data reduction and calibration procedures, the data set was cleaned from temporally occurring background caused by soft proton flares. The resulting observation time amounts to $14.4 \mathrm{ks}$ of useful data. Figure 4 shows an adaptively smoothed count map of the region surrounding HESS J1745-303 using events above $0.5 \mathrm{keV}$ detected with either the MOS 1 or MOS 2 detector of XMM-Newton. The white contours indicate the HESS VHE gamma-ray significance contours at $4 \sigma, 5 \sigma$ and $6 \sigma$. Six X-ray sources are apparent (labeled in red), as determined by the standard XMM-Newton source detection algorithm emldetect in the energy band from $0.5-10 \mathrm{keV}$, as well as in sub-intervals from $0.5-2 \mathrm{keV}, 2.0-4.5 \mathrm{keV}$ and from 4.5-10 keV. Table 3 summarizes the sources detected above $0.5 \mathrm{keV}$. For all these sources the algorithm also attempts 
Table 3. Sources detected using the detection algorithm emldetect. The parameters given here are for the energy range between 0.5 and $10 \mathrm{keV}$. Column 2: name recommended by the XMM-Newton SOC and the IAU for source detections. Columns 3 and 4: J2000.0 coordinates. Units of right ascension are hours, minutes, and seconds, and units of declination are degrees, arc-minutes, and arc-seconds. Column 5: error on the source position in arc-seconds. Column 6: number of counts in EMOS1 and EMOS2 within a 10" integration region using events above $0.5 \mathrm{keV}$. Column 7: statistical significance of the detection derived with emldetect. Column 8: source flux above $0.5 \mathrm{keV} \mathrm{in} 10^{-14} \mathrm{erg} \mathrm{cm}^{-2} \mathrm{~s}^{-1}$.

\begin{tabular}{c|c|ccc|ccc}
\hline \hline $\begin{array}{c}\text { Id } \\
(1)\end{array}$ & $\begin{array}{c}\text { XMMU J } \\
(2)\end{array}$ & $\begin{array}{c}\mathrm{RA}_{2000} \\
(3)\end{array}$ & $\begin{array}{c}\text { Dec }_{2000} \\
(4)\end{array}$ & $\begin{array}{c}\text { 2D Error } \\
(5)\end{array}$ & $\begin{array}{c}\text { Counts } \\
(6)\end{array}$ & $\begin{array}{c}\text { Significance } \\
(7)\end{array}$ & $\begin{array}{c}\text { Flux } \times 10^{-14} \\
(8)\end{array}$ \\
\hline 1 & $174434.5-301522$ & $17^{\mathrm{h}} 44^{\mathrm{m}} 34.47^{\mathrm{s}}$ & $-30^{\circ} 15^{\prime} 21.9^{\prime \prime}$ & 0.6 & 280 & 14.3 & $10.4 \pm 0.7$ \\
2 & $174441.3-301648$ & $17^{\mathrm{h}} 44^{\mathrm{m}} 41.27^{\mathrm{s}}$ & $-30^{\circ} 16^{\prime} 47.8^{\prime \prime}$ & 0.6 & 209 & 12.0 & $8.4 \pm 0.7$ \\
3 & $174437.6-301812$ & $17^{\mathrm{h}} 44^{\mathrm{m}} 37.55^{\mathrm{s}}$ & $-30^{\circ} 18^{\prime} 12.3^{\prime \prime}$ & 1.3 & 51 & 5.2 & $2.1 \pm 0.4$ \\
4 & $174425.3-302236$ & $17^{\mathrm{h}} 44^{\mathrm{m}} 25.30^{\mathrm{s}}$ & $-30^{\circ} 22^{\prime} 36.1^{\prime \prime}$ & 1.3 & 70 & 7.0 & $3.2 \pm 0.4$ \\
5 & $174458.6-301743$ & $17^{\mathrm{h}} 44^{\mathrm{m}} 58.59^{\mathrm{s}}$ & $-30^{\circ} 17^{\prime} 43.2^{\prime \prime}$ & 0.9 & 95 & 7.5 & $5.2 \pm 0.7$ \\
6 & $174351.0-301709$ & $17^{\mathrm{h}} 43^{\mathrm{m}} 51.03^{\mathrm{s}}$ & $-30^{\circ} 17^{\prime} 09.0^{\prime \prime}$ & 1.0 & 76 & 6.9 & $4.4 \pm 0.6$ \\
\hline
\end{tabular}

to determine a source extension by fitting a Gaussian model to the data. All six sources were found to be consistent with a point-source. Several of these X-ray sources coincide with stars known in the optical (shown in green in Fig. 4). Sources 1, 2, and 5 were found to be positionally coincident with HST optical guide stars as shown in Fig. 4, source 3 was found to coincide with an M3-star (RHI84 10-499) and for sources 4 and 6 a catalog search did not yield any obvious counterparts in other wavebands. As all of the detected X-ray sources are point-like and rather faint with fluxes around or below $10^{-14} \mathrm{erg} \mathrm{cm}^{-2} \mathrm{~s}^{-1}$, assuming a similar energy flux in X-rays and gamma rays, it seems unlikely that any of these sources are connected to the bright extended VHE gamma-ray source HESS J1745-303, which is extended and has an energy flux of $(2.00 \pm 0.18) \times$ $10^{-11} \mathrm{erg} \mathrm{cm}^{-2} \mathrm{~s}^{-1}$ above $200 \mathrm{GeV}$. In addition to the search for point-sources an analysis sensitive to diffuse X-ray emission has been performed. To that end, sources detected above a significance level of $3 \sigma$ were excluded from the raw counts map and the exposure map. The maps were then smoothed with a Gaussian of width $0.01^{\circ}$ and the ratio taken to produce the resulting smoothed, exposure-corrected counts map as shown in Fig. 5. Diffuse emission at a level similar to or above the level of the detected point-sources should show up in this method. However, no sign of such a diffuse emission is detected in the whole field of view and we derive a $99 \%$ confidence limit on the flux level of the diffuse emission in region $\mathrm{A}$ of $4.5 \times$ $10^{-13} \mathrm{erg} \mathrm{cm}^{-2} \mathrm{~s}^{-1}$, using the rest of the field-of-view to determine the background, and $7.1 \times 10^{-13} \mathrm{erg} \mathrm{cm}^{-2} \mathrm{~s}^{-1}$ using only a strip to the west of region $\mathrm{A}$ as background.

\section{Possible associations}

To look for possible associations, standard catalogs of sources thought to be associated with VHE emission were searched, including high-spin-down flux ${ }^{1}$ pulsars (Manchester et al. 2005), SNRs (Green 2004), Wolf-Rayet stars (van der Hucht 2001), high-mass X-ray binaries (Liu et al. 2006), INTEGRAL sources (Bird et al. 2007), and HII regions. Additionally, public survey data from ROSAT (Voges et al. 2000), ASCA (Tanaka et al. 1994), and the VLA (LaRosa et al. 2000) were searched for possible un-cataloged counterparts. The most likely candidates found in this search are discussed here.

\footnotetext{
${ }^{1}$ High spin-down flux $\left(\dot{E} / D^{2}\right)$ pulsars are thought to be likely VHE emission candidates if the conversion efficiency from spin-down power to VHE emission is around 1\% (e.g. Carrigan et al. 2007).
}

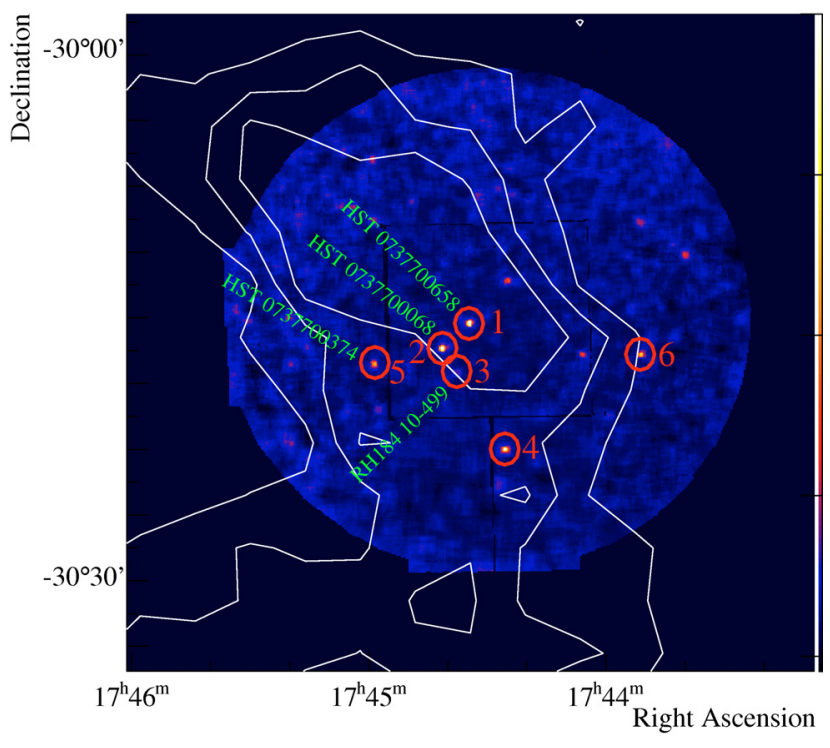

Fig. 4. Point sources detected within the XMM-Newton exposure (see Table 3 for detailed information). The HESS 4, 5, $6 \sigma$ significance contours from Fig. 1 are overlaid in white. Apparently associated stars are labeled.

\subsection{G359.1-0.5 and molecular clouds}

G359.1-0.5 was identified as an SNR by Downes et al. (1979) using the Westerbork Synthesis Radio Telescope and the Bonn 100-m telescope. VLA observations (Uchida et al. 1992a) confirmed the presence of the non-thermal shell and HI absorption showed that its probable location is within a few hundred parsecs of the Galactic center. At an estimated distance of $7.6 \mathrm{kpc}$, the $12^{\prime}$ radius of the remnant corresponds to $26.5 \mathrm{pc}$, with an estimated age of $\geq 10^{4} \mathrm{yr}$ (a middle aged SNR). Observations of the ${ }^{12} \mathrm{CO}$ emission line $(J=1 \rightarrow 0)$ with the Bell Laboratories telescope (Uchida et al. 1992b) revealed a dense ring of matter surrounding the shell. The radial velocity dispersion of this super-shell between -60 and $-190 \mathrm{~km} \mathrm{~s}^{-1}$ agrees well with a location of the remnant close to the Galactic center. This supershell could have been produced by the combined stellar winds of 200 O-type stars concentrated in this region, which is very probably the birth place of the remnant (Uchida et al. 1992b). X-ray observations of the remnant with ASCA showed no evidence for a shell in the energy range $3.2-10.0 \mathrm{keV}$, detecting only thermal diffuse emission from the central region (Bamba et al. 2000). 


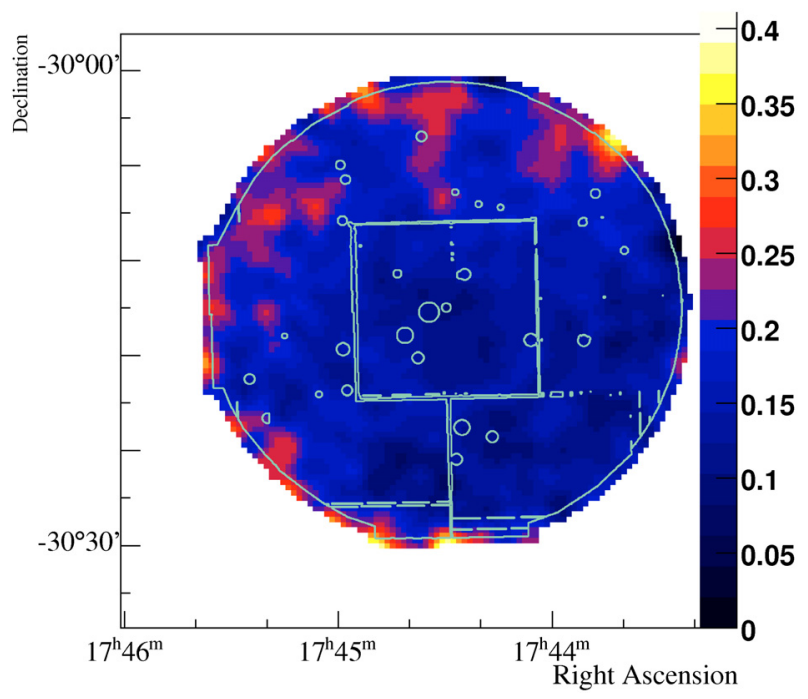

Fig. 5. Exposure corrected X-ray image smoothed with a $0.01^{\circ}$ Gaussian filter. All point sources above $3 \sigma$ and the borders of each detector chip (shown by the contour) have been excluded before smoothing. No evidence for diffuse emission is seen.

Uchida et al. (1992b) observed a correlation in intensity between the non-thermal radio emission of the remnant and the $\mathrm{CO}$ emission and suggested an association of the two. There are no sharp gradients observed in the velocity distribution, the absence of which could be explained by the presence of magnetic precursors or by a previous acceleration of the shell by stellar winds or old supernovae. Another indication of the interaction of the remnant with the surrounding medium is the presence of maser emission spots near the edge of the shell, revealed by VLA observations (Yusef-Zadeh et al. 1995). OH masers at $1720 \mathrm{MHz}$ are believed to be produced by collisional pumping behind shocks and are therefore a good indicator of shocked clouds (Elitzur 1976). Although the mean velocity of the masers at around $-5 \mathrm{~km} \mathrm{~s}^{-1}$ is significantly shifted from the velocity range of the ${ }^{12} \mathrm{CO}$ shell $\left([-60,-190] \mathrm{km} \mathrm{s}^{-1}\right)$, a random coincidence of the shell with the maser positions seems unlikely. Yusef-Zadeh et al. (1995) discussed this velocity discrepancy: the two velocities could be reconciled if the shocked cloud was carried away by the shock itself, which could have redirected the radial velocity component. The specific conditions required to allow a population inversion (and thus maser emission) may also explain this discrepancy. Moreover, the association between the $\mathrm{OH}$ masers, the SNR and the ${ }^{12} \mathrm{CO}$ cloud is supported by the fact that the maser distribution shows a good correlation with the ${ }^{12} \mathrm{CO}$ emission maximum and the non-thermal radio emission from the western part of the shell.

We have used ${ }^{12} \mathrm{CO}(J=1 \rightarrow 0)$ data from the Cerro Tololo Inter-American Observatory (Chile) to map the matter distribution in the western part of the remnant (Bitran et al. 1997). Figure 6 is the ${ }^{12} \mathrm{CO}$ map integrated in velocity between $-100 \mathrm{~km} \mathrm{~s}^{-1}$ and $-60 \mathrm{~km} \mathrm{~s}^{-1}$. This velocity range corresponds to the western part of the super-shell surrounding the remnant and is potentially associated with the remnant. Two overlapping clouds are present in this region. The cloud shown in Fig. 6 contains a component that is partially coincident with HESS J1745-303.

Due to the relatively high magnetic field, $(0.2-0.6 \mathrm{mG}$, as measured from the Zeeman splitting of maser lines

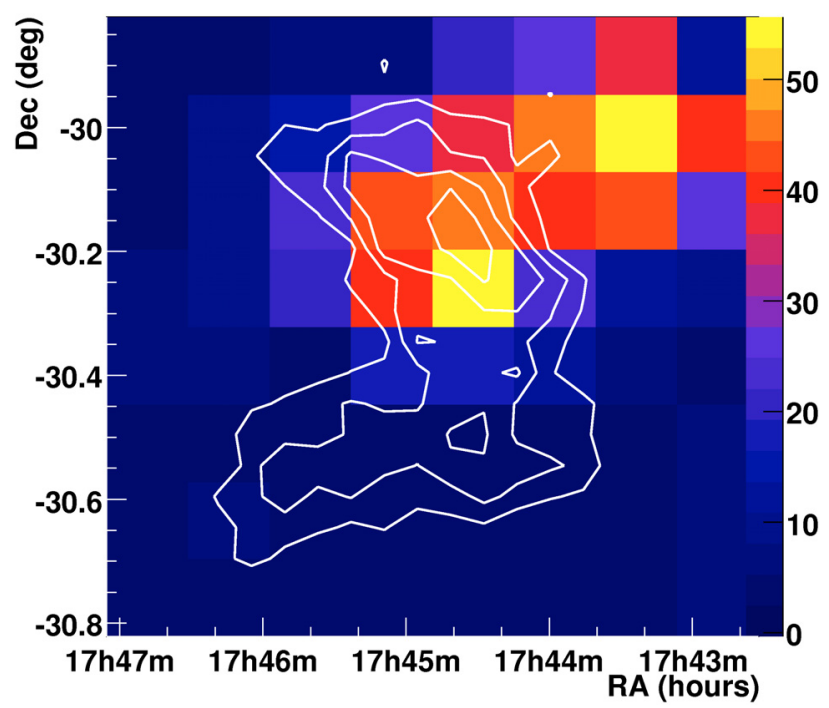

Fig. 6. Velocity-integrated (-100 to $-60 \mathrm{~km} \mathrm{~s}^{-1}$ ) map of ${ }^{12} \mathrm{CO}$ (Bitran et al. 1997) emission showing the molecular cloud coincident with the shell of G359.1-0.5, with overlaid HESS $4 \sigma$ to $7 \sigma$ significance contours.

(Robinson et al. 1996), the cooling time of ultra-relativistic electrons would be much shorter than the age of the remnant, and therefore an electron production scenario seems unlikely as an explanation of the VHE emission. A possible explanation is that a part of the VHE gamma-ray source comes instead from hadronic cosmic-ray interactions in this cloud, producing neutral pions that decay into two gamma rays. Assuming a value of the ratio $X=N_{\mathrm{H}_{2}} / W_{\mathrm{CO}}$ of $1.8 \times 10^{20} \mathrm{~cm}^{-2} \mathrm{~K}^{-1} \mathrm{~km}^{-1} \mathrm{~s}^{-1}$ (Dame et al. 2001), we estimate the $\mathrm{H}_{2}$ mass in this cloud to be $5 \times 10^{4} M_{\odot}$ with a density of $5 \times 10^{3} \mathrm{~cm}^{-3}$, assuming spherical symmetry. The value of the ratio $X$ used is a mean galactic value which may not be relevant for the inner part of the galaxy, therefore the cloud mass may be overestimated. A spectral analysis of the part of HESS J1745-303 coincident with the cloud (region A) indicates that approximately $15 \%$ of the observed flux comes from this region (see Table 2).

Since hadronic interactions lead to the production of gamma rays with energies typically a factor of 10 below the primary energy, the energy of interacting protons $(W)$ required to generate the observed flux of VHE $\gamma$-rays between $300 \mathrm{GeV}$ and $40 \mathrm{TeV}$ can be estimated in the corresponding energy range of approximately $3-400 \mathrm{TeV}$ to be: $W=t_{\mathrm{pp} \rightarrow \pi^{0}} \cdot L_{\gamma}$, where $t_{\mathrm{pp} \rightarrow \pi^{0}}$ is the characteristic cooling time of protons through the $\pi^{0}$ production channel and $L_{\gamma}$ is the gamma-ray luminosity between $300 \mathrm{GeV}$ and $40 \mathrm{TeV}$ (Aharonian \& Atoyan 1996). Assuming that the proton energy distribution follows a power law with the same index as the gamma rays over the full relativistic range, we can extrapolate this distribution down to $1 \mathrm{GeV}$. Assuming also that the accelerated proton density is uniform in the whole remnant, it corresponds to a fraction of $\sim 32 \%$ of the mechanical explosion energy of $10^{51} \mathrm{erg}$ of the remnant. This estimate suffers from large uncertainties, mainly from the cloud mass estimation, the fraction of HESS J1745-303 involved in this association, and the explosion energy. However, it is interesting that we obtain an estimate that is comparable to the theoretical espectation of $\sim 10 \%$. 


\subsection{EG J1744-3011}

Most of the emission associated with HESS J1745-303 lies within the $95 \%$ error-circle of the unidentified EGRET source 3EG J1744-3011 (marked with a dotted circle in Fig. 1), which has an integral flux in the $100 \mathrm{MeV}-10 \mathrm{GeV}$ energy band of $(63.9 \pm 7.1) \times 10^{-8} \mathrm{~cm}^{-2} \mathrm{~s}^{-1}$ with a photon index of $\Gamma_{\text {EGRET }}=$ $2.17 \pm 0.08$ (Hartman et al. 1999). Extrapolating this flux to the VHE range, we find an expected integral flux in the range $1-10 \mathrm{TeV}$ of $\left(3_{-1}^{+4}\right) \times 10^{-11} \mathrm{~cm}^{-2} \mathrm{~s}^{-1}$, which is higher than the integral flux observed by HESS over the same range (see Fig. 2). Fitting a power law with an exponential cutoff to the HESS spectrum combined with the EGRET flux point, we find a cutoff energy of $\sim(3.0 \pm 1.5) \mathrm{TeV}$.

A study of the long-term variability of EGRET sources made by Torres et al. (2001) shows that 3EG J1744-3011 is variable (on year timescales), with an average statistical index of variability over $3 \sigma$ higher than that expected from pulsars (which are considered a non-variable source used as a reference for systematic variability of the instrument). If the EGRET source is truly variable, it is unlikely to be associated with the extended emission seen in HESS J1745-303, where no variability would be expected.

\subsection{PSR B1742-30, PSR J1747-2958, SLX 1744-299}

Two cataloged pulsars lie within or near HESS J1745-303: PSR B1742-30 and PSR J1747-2958 (see Fig. 1). Energetic pulsars driving pulsar wind nebulae are known to produce VHE emission that may be asymmetric or offset from the pulsar position (e.g. Aharonian et al. 2006b,f, 2005d,b). In several cases, the PWN candidate has been first identified in the VHE energy range and subsequently confirmed with X-ray measurements (e.g. Aharonian et al. 2007a; Hinton et al. 2007).

PSR B1742-30 (also known as PSR J1745-3040) is a rather old pulsar (546 kyr) located near the southern edge of the region of significant emission in HESS J1745-303, and has a spindown flux $\dot{E} / D^{2}=2 \times 10^{33} \mathrm{erg} \mathrm{s}^{-1} \mathrm{kpc}^{-2}$ (Manchester et al. 2005), requiring a conversion efficiency from rotational kinetic energy to gamma-ray emission of approximately $32 \%$ to produce the entire VHE emission. Such high apparent efficiencies are possible if the spin-down flux was much higher in the past and the particle cooling times are comparable to or much shorter than the pulsar age. If PSR B1742-30 powers only the fraction of HESS J1745-303 enclosed by region B, then the required conversion efficiency would be only $2 \%$, which is not unreasonable compared with other known VHE PWNe.

PSR J1747-2958 is located approximately a half-degree east of HESS J1745-303 and is associated with the bright X-ray and radio feature G359.23-0.82, also known as "the Mouse" (Gaensler et al. 2004) (seen in the radio image in Fig. 1), which is believed to be a bow-shock PWN with a trailing tail caused by the reverse termination shock. Given the proper velocity, estimated to be $\sim 600 \mathrm{~km} \mathrm{~s}^{-1}$ (Gaensler et al. 2004), distance $D \simeq$ $2.08 \mathrm{kpc}$ and age (25.5 kyr) (Manchester et al. 2005), one can extrapolate that the pulsar would have moved approximately $0.43^{\circ}$ from its original position, placing it close to HESS J1745-303 if the direction of motion is along the tail of the "Mouse" (see dotted line in Fig. 1, Bottom). The relatively high spin-down flux of PSR J1747-2958 $\left(\dot{E} / D^{2}=4 \times 10^{35} \mathrm{erg} \mathrm{s}^{-1} \mathrm{kpc}^{-2}\right)$ would imply a $0.2 \%$ conversion efficiency to explain the entire VHE emission, or $0.02 \%$ for only region $A$. In this case, the PWN would have to be extremely offset and asymmetric.
Located near PSR J1747-2958 are the ultra-compact X-ray binary SLX 1744-299 (in 't Zand et al. 2007), and the X-ray burster 1744-300 (Skinner et al. 1990), which are not generally expected to produce offset or extended emission and are thus not considered probable counterparts to HESS J1745-303.

\section{Discussion}

Due to the positional coincidence and plausible energetics, at least part of the emission of HESS J1745-303 (in the region labeled $A$ in Fig. 1) may well be associated with the interaction of the shell of G359.1-0.5 with a molecular cloud. This scenario fits particularly well within the context of theoretical predictions for VHE gamma-ray emission from SNRs embedded in dense media. Gabici \& Aharonian (2007) show that for a SNR of approximately the same age as G359.1-0.5 in this context, one would expect significant $\mathrm{TeV}$ emission that peaks around $1 \mathrm{TeV}$. Assuming a similar supernova energy output of $10^{51} \mathrm{erg}$ and scaling their theoretical model for a cloud at $30 \mathrm{pc}$ away from an SNR shell by $M_{\mathrm{cl}} / D^{2}$, where $M_{\mathrm{cl}}$ is the mass of the cloud near G359.1-0.5 and $D$ is its distance, we find that the predicted flux at $1 \mathrm{TeV}$ of $2.8 \times 10^{-12} \mathrm{TeV}^{-1} \mathrm{~cm}^{-2} \mathrm{~s}^{-1}$ matches well with the HESS J1745-303 emission around region $A, F_{A}(1 \mathrm{TeV})=(4.4 \pm 0.5) \times 10^{-12} \mathrm{TeV}^{-1} \mathrm{~cm}^{-2} \mathrm{~s}^{-1}$, as does the soft $\Gamma \simeq 3.0$ spectral index.

However, since the VHE emission extends beyond region $A$ (and the dense target material does not), the SNR/molecularcloud scenario is not sufficient for describing the entire VHE source. Since there is as yet no statistically significant separation between the various parts of the emission region, an explanation for the entire source remains complicated and the possibility of source confusion still remains. In particular, PSR B1742-30 is energetic enough to power a PWN in the part of HESS J1745-303 surrounding it (region B), and though an unlikely candidate due to its significant offset, PSR J1747-2958 is in principle powerful enough to power the entire VHE source. The lack of significant spectral variability across the emission region further complicates the identification of counterparts. It is important to note that many VHE sources and source classes have spectral indices in the range 2.1-2.7, therefore given the statistics, it would be likely impossible to disentangle a superposition of three randomly chosen VHE sources from their spectra alone.

Furthermore, the lack of a significant extended source in the XMM-Newton data is not without precedent - several other TeV sources, such as HESS-J1303-631 (Aharonian et al. 2005c), other unidentified TeV sources (Aharonian et al. 2008), have so far no identified X-ray counterparts The lack of nonthermal X-ray or radio emission combined with the relatively high magnetic field around G359.1-0.5 further supports the hadronic scenario for VHE gamma-ray production for the region associated with the interaction of the SNR shock with the target material in the molecular cloud. However, even in a hadronic scenario, some longer-wavelength emission would be expected due to secondary electrons produced in the interactions.

An association of 3EG J1744-3011 with part or all of HESS J1745-303 is also plausible from an energetic standpoint (e.g. Funk et al. 2007), however since the size of the EGRET error circle is larger than the VHE emission, the position may not correspond with the VHE source and further localization is not currently possible. Aharonian \& Atoyan (1996) show that the observed gamma-ray flux from a hadronic source is proportional to $E_{\gamma}^{-\left(\Gamma_{p}+\delta\right)}$, where $\Gamma_{\mathrm{p}}$ is the proton index at the source and $\delta$ is 
the index of the diffusion coefficient (typically 0.3-0.6), allowing spectra that are quite soft in the TeV energy range, and which may be different from the slopes in other energy bands (Torres et al. 2003). Therefore for an $E_{\mathrm{p}}^{-2.0}$ source spectrum, it is possible to reproduce both the hard $(\Gamma=2.17)$ EGRET spectrum and the softer $\Gamma=2.71 \pm 0.11_{\text {stat }} \pm 0.2_{\text {sys }} \mathrm{TeV}$ spectrum. If we assume 3EG J1744-3011 is associated only with the SNR/molecularcloud interaction, then the observed flux is significantly higher than that predicted by the Gabici \& Aharonian (2007) model, however given the uncertainties in position, the EGRET flux may also contain contributions from the SNR shell itself (where gamma rays may be produced by e.g. inverse-Compton scattering of lower energy photons by high-energy electrons accelerated in the shock), or from other sources in the region, which could explain this.

Further multi-wavelength observations in X-rays and $\mathrm{GeV}$ gamma rays (e.g. from observatories such as XMM, Suzaku and GLAST) of the entire region spanned by HESS J1745-303 as well as deeper VHE exposures will be needed to disentangle the emission possibilities and to find more definitive counterparts.

Acknowledgements. The support of the Namibian authorities and of the University of Namibia in facilitating the construction and operation of HESS is gratefully acknowledged, as is the support by the German Ministry for Education and Research (BMBF), the Max Planck Society, the French Ministry for Research, the CNRS-IN2P3 and the Astroparticle Interdisciplinary Programme of the CNRS, the UK Science and Technology Facilities Council (STFC), the IPNP of the Charles University, the Polish Ministry of Science and Higher Education, the South African Department of Science and Technology and National Research Foundation, and by the University of Namibia. We appreciate the excellent work of the technical support staff in Berlin, Durham, Hamburg, Heidelberg, Palaiseau, Paris, Saclay, and in Namibia in the construction and operation of the equipment.

We would like to further thank Thomas Dame for providing us with the CO data. This research has made use of the SIMBAD database, operated at CDS, Strasbourg, France and the ROSAT Data Archive of the Max-Planck-Institut für extraterrestrische Physik (MPE) at Garching, Germany.

\section{References}

Aharonian, F. A., \& Atoyan, A. M. 1996, A\&A, 309, 917

Aharonian, F. A., Drury, L. O., \& Voelk, H. J. 1994, A\&A, 285, 645

Aharonian, F., Akhperjanian, A. G., Aye, K.-M., et al. 2004, A\&A, 425, L13

Aharonian, F., Akhperjanian, A. G., Aye, K.-M., et al. 2005a, A\&A, 432, L25

Aharonian, F., Akhperjanian, A. G., Aye, K.-M., et al. 2005b, A\&A, 435, L17

Aharonian, F., Akhperjanian, A. G., Aye, K.-M., et al. 2005c, A\&A, 439, 1013

Aharonian, F. A., Akhperjanian, A. G., Bazer-Bachi, A. R., et al. 2005d, A\&A, 442, L25

Aharonian, F., Akhperjanian, A. G., Bazer-Bachi, A. R., et al. 2006a, A\&A, 457, 899

Aharonian, F., Akhperjanian, A. G., Bazer-Bachi, A. R., et al. 2006b, A\&A, 448, L43

Aharonian, F., Akhperjanian, A. G., Bazer-Bachi, A. R., et al. 2006c, Nature, 439, 695

Aharonian, F., Akhperjanian, A. G., Bazer-Bachi, A. R., et al. 2006d, ApJ, 636, 777

Aharonian, F., Akhperjanian, A. G., Bazer-Bachi, A. R., et al. 2006e, A\&A, 449, 223

Aharonian, F., Akhperjanian, A. G., Bazer-Bachi, A. R., et al. 2006f, A\&A, 456, 245

Aharonian, F., Akhperjanian, A. G., Bazer-Bachi, A. R., et al. 2007a, A\&A, 472, 489

Aharonian, F., Akhperjanian, A. G., Bazer-Bachi, A. R., et al. 2007b, A\&A, 467, 1075

Aharonian, F., Akhperjanian, A. G., Barres de Almeida, U., et al. 2008, A\&A, 477,353

Bamba, A., Yokogawa, J., Sakano, M., \& Koyama, K. 2000, PASJ, 52, 259

Berge, D., Funk, S., \& Hinton, J. 2007, A\&A, 466, 1219
Bernlöhr, K., Carrol, O., Cornils, R., et al. 2003, Astropart. Phys., 20, 111 Bird, A. J., Malizia, A., Bazzano, A., et al. 2007, ApJS, 170, 175 Bitran, M., Alvarez, H., Bronfman, L., May, J., \& Thaddeus, P. 1997, A\&AS, 125,99

Carrigan, S., Hinton, J. A., Hofmann, W., et al. 2007, ArXiv e-prints, 709 Dame, T. M., Hartmann, D., \& Thaddeus, P. 2001, ApJ, 547, 792

Daum, A., Hermann, G., Hess, M., et al. 1997, Astropart. Phys., 8, 1 de Naurois, M. 2005, in Proc. of the Conf., Towards a Network of Atmospheric Cherenkov Detectors VII, Palaiseau, France

[arXiv: astro-ph/0607247]

Downes, D., Goss, W. M., Schwarz, U. J., \& Wouterloot, J. G. A. 1979, A\&AS, 35,1

Elitzur, M. 1976, ApJ, 203, 124

Funk, S., Reimer, O., Torres, D. F., \& Hinton, J. A. 2007, ArXiv e-prints, 710 Gabici, S., \& Aharonian, F. A. 2007, ApJ, 665, L131

Gaensler, B. M., van der Swaluw, E., Camilo, F., et al. 2004, ApJ, 616, 383 Green, D. A. 2004, Bull. Astron. Soc. India, 32, 335

Hartman, R. C., Bertsch, D. L., Bloom, S. D., et al. 1999, ApJS, 123, 79 Hillas, A. M. 1985, in Int. Cosmic Ray Conf. 3, ed. F. C. Jones, 445

Hillas, A. M. 1996, Space Sci. Rev., 75, 17

Hinton, J. A., \& Aharonian, F. A. 2007, ApJ, 657, 302

Hinton, J. A., Funk, S., Carrigan, S., et al. 2007, ArXiv e-prints, 710

in 't Zand, J. J. M., Jonker, P. G., \& Markwardt, C. B. 2007, A\&A, 465, 953

Kosack, K., Badran, H. M., Bond, I. H., et al. 2004, ApJ, 608, L97

LaRosa, T. N., Kassim, N. E., Lazio, T. J. W., \& Hyman, S. D. 2000, AJ, 119, 207

Li, T.-P., \& Ma, Y.-Q. 1983, ApJ, 272, 317

Liu, Q. Z., van Paradijs, J., \& van den Heuvel, E. P. J. 2006, A\&A, 455, 1165

Manchester, R. N., Hobbs, G. B., Teoh, A., \& Hobbs, M. 2005, AJ, 129, 1993

Piron, F., Djannati-Atai, A., Punch, M., et al. 2001, A\&A, 374, 895

Robinson, B., Yusef-Zadeh, F., \& Roberts, D. 1996, in BAAS, 28, 948

Skinner, G. K., Foster, A. J., Willmore, A. P., \& Eyles, C. J. 1990, MNRAS, 243 , 72

Snowden, S. L., Collier, M. R., \& Kuntz, K. D. 2004, ApJ, 610, 1182

Tanaka, Y., Inoue, H., \& Holt, S. S. 1994, PASJ, 46, L37

Torres, D. F., Romero, G. E., Combi, J. A., et al. 2001, A\&A, 370, 468

Torres, D. F., Romero, G. E., Dame, T. M., Combi, J. A., \& Butt, Y. M. 2003, Phys. Rep., 382, 303

Tsuchiya, K., Enomoto, R., Ksenofontov, L. T., et al. 2004, ApJ, 606, L115

Uchida, K., Morris, M., \& Yusef-Zadeh, F. 1992a, AJ, 104, 1533

Uchida, K. I., Morris, M., Bally, J., Pound, M., \& Yusef-Zadeh, F. 1992b, ApJ, 398,128

van der Hucht, K. A. 2001, VizieR Online Data Catalog, 3215

Voges, W., Aschenbach, B., Boller, T., et al. 2000, IAU Circ., 7432, 3

Weekes, T. C. 1996, Space Sci. Rev., 75, 1

Yusef-Zadeh, F., Uchida, K. I., \& Roberts, D. 1995, Science, 270, 1801

1 Max-Planck-Institut für Kernphysik, PO Box 103980, 69029 Heidelberg, Germany

e-mail: Karl.Kosack@mpi-hd.mpg.de

2 Yerevan Physics Institute, 2 Alikhanian Brothers St., 375036

Yerevan, Armenia

3 Centre d'Étude Spatiale des Rayonnements, CNRS/UPS, 9 Av. du Colonel Roche, BP 4346, 31029 Toulouse Cedex 4, France

4 Universität Hamburg, Institut für Experimentalphysik, Luruper Chaussee 149, 22761 Hamburg, Germany

5 Institut für Physik, Humboldt-Universität zu Berlin, Newtonstr. 15, 12489 Berlin, Germany

6 LUTH, Observatoire de Paris, CNRS, Université Paris Diderot, 5 place Jules Janssen, 92190 Meudon, France

7 DAPNIA/DSM/CEA, CE Saclay, 91191 Gif-sur-Yvette Cedex, France

${ }^{8}$ University of Durham, Department of Physics, South Road, Durham DH1 3LE, UK

9 Unit for Space Physics, North-West University, Potchefstroom 2520, South Africa

${ }^{10}$ Laboratoire Leprince-Ringuet, École Polytechnique, CNRS/ IN2P3, 91128 Palaiseau, France

11 Laboratoire d'Annecy-le-Vieux de Physique des Particules, CNRS/IN2P3, 9 chemin de Bellevue, BP 110, 74941 Annecy-leVieux Cedex, France

12 Astroparticule et Cosmologie (APC), CNRS, Université Paris 7 Denis Diderot, 10 rue Alice Domon et Léonie Duquet, 75205 Paris Cedex 13; UMR 7164 (CNRS, Université Paris VII, CEA, Observatoire de Paris), France 
13 Dublin Institute for Advanced Studies, 5 Merrion Square, Dublin 2, Ireland

14 Landessternwarte, Universität Heidelberg, Königstuhl, 69117 Heidelberg, Germany

${ }_{15}$ Laboratoire de Physique Théorique et Astroparticules, CNRS/IN2P3, Université Montpellier II, CC 70, Place Eugène Bataillon, 34095 Montpellier Cedex 5, France

e-mail: Armand.Fiasson@LPTA.in2p3.fr

${ }^{16}$ Universität Erlangen-Nürnberg, Physikalisches Institut, ErwinRommel-Str. 1, 91058 Erlangen, Germany

${ }_{17}$ Laboratoire d'Astrophysique de Grenoble, INSU/CNRS, Université Joseph Fourier, BP 53, 38041 Grenoble Cedex 9, France

${ }^{18}$ Institut für Astronomie und Astrophysik, Universität Tübingen, Sand 1, 72076 Tübingen, Germany

19 LPNHE, Université Pierre et Marie Curie Paris 6, Université Denis Diderot Paris 7, CNRS/IN2P3, 4 place Jussieu, 75252 Paris Cedex 5, France
20 Institute of Particle and Nuclear Physics, Charles University, V Holesovickach 2, 18000 Prague 8, Czech Republic

21 Institut für Theoretische Physik, Lehrstuhl IV: Weltraum und Astrophysik, Ruhr-Universität Bochum, 44780 Bochum, Germany

22 University of Namibia, Private Bag 13301, Windhoek, Namibia

23 Obserwatorium Astronomiczne, Uniwersytet Jagielloński, Kraków, Poland

${ }^{24}$ Nicolaus Copernicus Astronomical Center, Warsaw, Poland

25 School of Physics \& Astronomy, University of Leeds, Leeds LS2 9JT, UK

${ }^{26}$ School of Chemistry \& Physics, University of Adelaide, Adelaide 5005, Australia

27 Toruń Centre for Astronomy, Nicolaus Copernicus University, Toruń, Poland

28 European Associated Laboratory for Gamma-Ray Astronomy, jointly supported by CNRS and MPG

${ }_{29}$ Kavli Institute for Particle Astrophysics and Cosmology, SLAC, 2575 Sand Hill Road, Menlo-Park, CA-94025, USA 